\title{
THE STATISTICAL CHARACTER OF PACKED-BED HEAT TRANSPORT PROPERTIES
}

\author{
R. J. WIJNGAARDEN ${ }^{\dagger}$ and K. R. WESTERTERP ${ }^{\ddagger}$ \\ Chemical Reaction Engineering Laboratories, Department of Chemical Engineering, Twente University, \\ PO Box 217, 7500 AE Enschede, The Netherlands
}

(Received 25 February 1991; accepted for publication 6 December 1991)

\begin{abstract}
Packed beds are essentially heterogeneous on a pellet scale. For random packed beds this heterogeneity causes a statistical character both on a pellet and bed scale. We discuss experimental results which deal with bed-scale statistics. For packed beds on a laboratory scale, the results indicate that the bedscale statistical behaviour introduces a spread up to a factor of three in correlations for the effective radial heat conductivity, $\lambda_{\text {eff }}$, and the heat transfer coefficient at the wall, $\alpha_{w}$. This spread of a factor of three roughly equals the spread between correlations available in the literature. For cooled tubular reactors the statistical spread in the behaviour of the individual tubes will have a large influence on reactor operation.
\end{abstract}

\section{INTRODUCTION}

Packed-bed properties are distributed both in space and in time. Distribution in time could lead to chaotic behaviour. Chaotic behaviour was studied by Kirillov et al. (1972), who have visualized the flow of gases and liquids in packed beds. Within the voids between the pellets, a flow-through zone exists in which the fluid flows along tortuous paths in the form of blending and separating streams. Further, a non-flow zone exists in the vicinity of the pellet contact points. A sharp separation boundary is formed between the two zones. This separation boundary is constantly being deformed by the existence of rotating and pulsating vortices in the non-flow zone. The pulsation frequency, $f$, is directly proportional to the local velocity of the streams and inversely proportional to the pellet size, so that the Strouhal number $S r=\varepsilon f d_{p} / v_{0} \approx 1 / 2$ for $20<R e<1000$. For packed beds, as used in the chemical industry, this means that the jets erupting from the voids between the pellets are pulsating and vibrating with frequencies from 10 to $100 \mathrm{~Hz}$. Thus, for industrial packed beds, this chaotic behaviour becomes important only if phenomena on a pellet scale with a characteristic time of 0.1 to $0.01 \mathrm{~s}$ play a role. Typically, characteristic times on a pellet scale for reaction or intra-particle diffusion lie in the order of 1 to $10 \mathrm{~s}$. Therefore, the mentioned chaotic behaviour will usually be unimportant.

Therefore, we will focus on statistical distributions of packed-bed properties in space. If the packing of the bed is random, the local porosity, and hence also the velocity and temperature, will, to a certain extent. be randomly distributed in space (see Fig. 1). Because of the mathematical difficulties in accounting for this random distribution in space, packed beds are usually

\footnotetext{
'Present address: Koninklijke/Shell-Laboratorium, Amsterdam, PO Box 3003, AA Amsterdam, The Netherlands.

tAuthor to whom correspondence should be addressed.
}

described by homogeneous or pseudo-homogeneous models, i.e. the solid phase and the gas phase are regarded as one single phase with average properties, like an average bulk porosity, an average gas velocity, and hence a smooth temperature profile. For a homogeneous model we can vary the average bed properties as a function of the place in the packed bed in any way we want, as long as that function is deterministic, e.g. we can choose a uniform porosity profile and neglect the wall effects, or we can account for wall effects and vary the porosity from unity, at the wall, to lower values further away from the wall (see Fig. 1).

Here we shall show that this statistical distribution in space can cause a statistical distribution of packedbed heat transport properties.

\section{TEMPERATURE PROFILES IN PACKED BEDS}

Part of the scatter in temperature measurements in packed beds arises from the fact that the packed bed is heterogeneous on a pellet scale, i.e. if a radial profile is followed at a single axial position, the local porosity is a Boolean variable (see Fig. 1). Another part arises from the fact that gas streams within the voids are directed in space in a statistical manner. As a result, measured temperature profiles also exhibit a certain amount of scatter (see Fig. 2).

This is confirmed by Cresswell (1986) who measured radial temperature profiles in a paked bed and found a $10^{\circ} \mathrm{C}$ spread between angular replicates for a total gradient of $40^{\circ} \mathrm{C}$. We measured the radial temperature profiles in a packed bed through which a cold gas (temperature $20^{\circ} \mathrm{C}$ ) flows and which is heated at the wall (temperature $60^{\circ} \mathrm{C}$ ). The results are indicated in Fig. 3. We repeated the temperature profile measurements several times; in between the experiments the packed bed was fluidized for a few seconds so that a new random packing was obtained. The thin lines in Fig. 3 represent the individual temperature profiles; the bold line gives the average temperature 


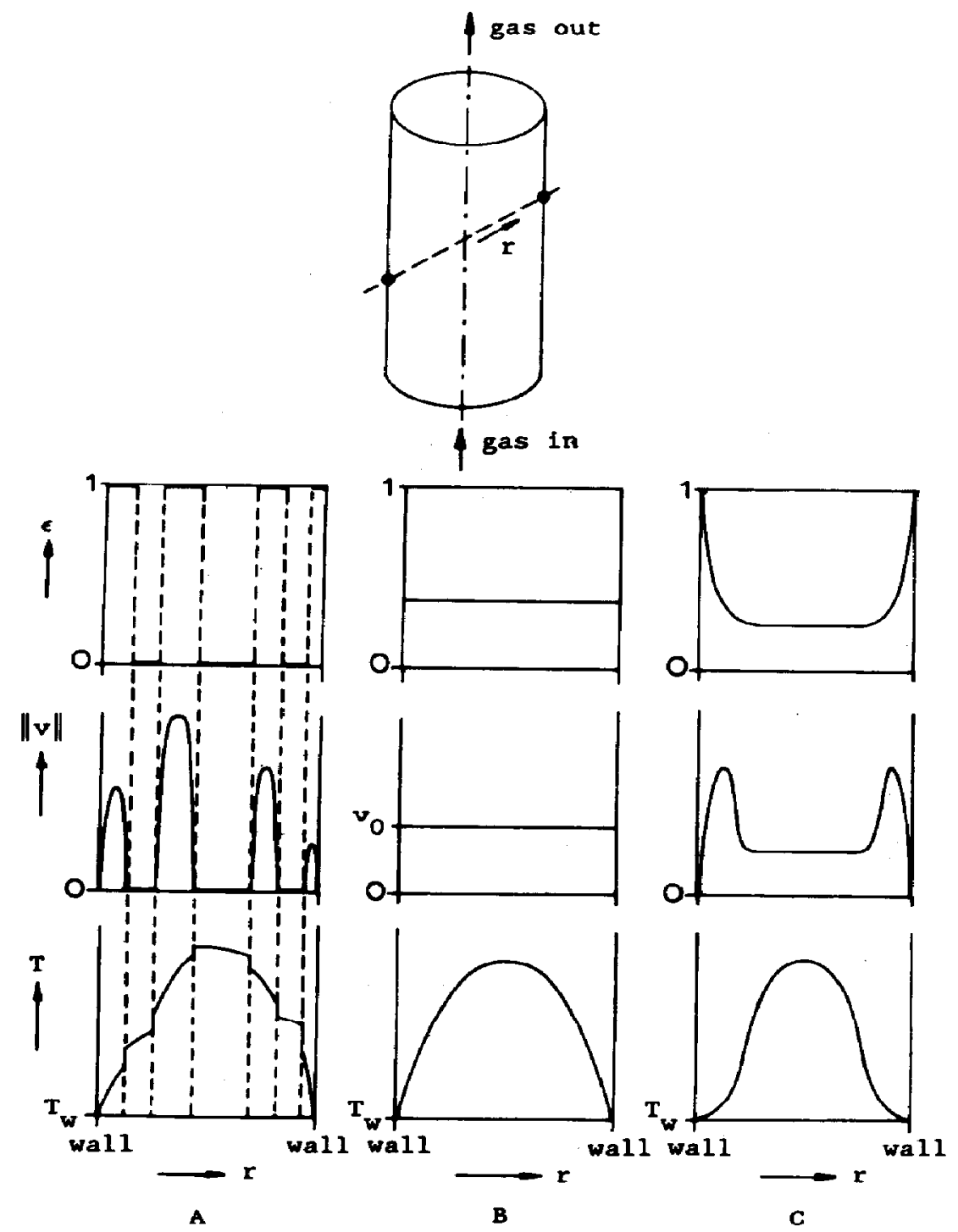

Fig. 1. Porosity, $\epsilon$, velocity norm, $\|v\|$, and temperature, $T$, vs the distance $r$ in a packed bed: (A) actual profiles, (B) homogeneous model without wall effect, (C) hormogeneous model with wall effect.

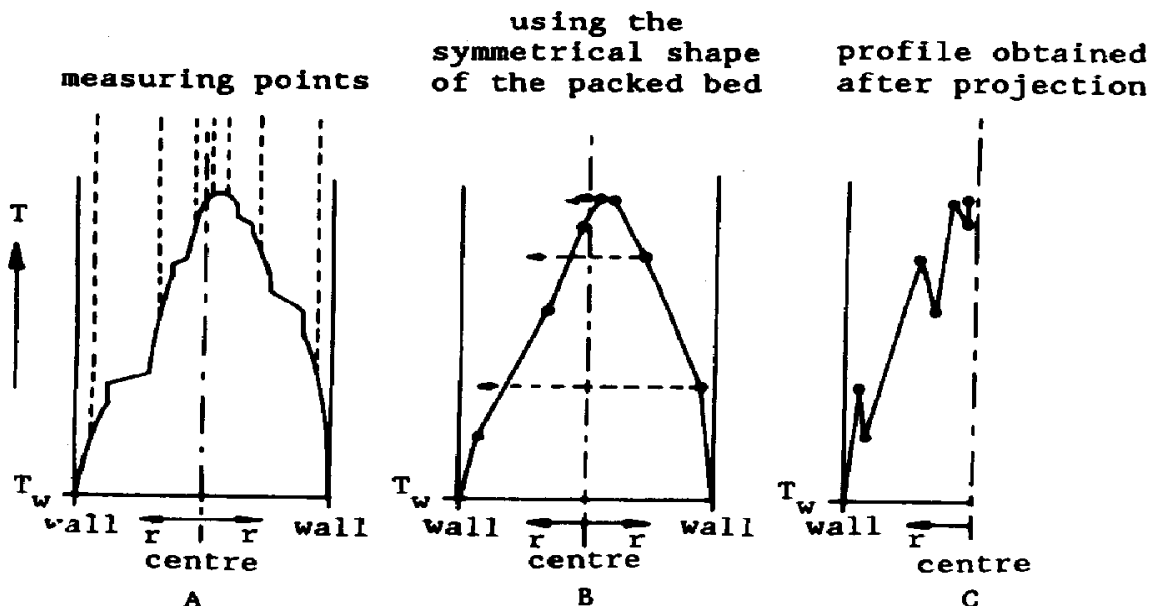

Fig. 2. Illustration of how scatter in a temperature profile is obtained: (A) actual profile and measuring points, (B) measured temperature profile, (C) profile which is obtained if the cylindrical symmetry of the packed bed is included. 


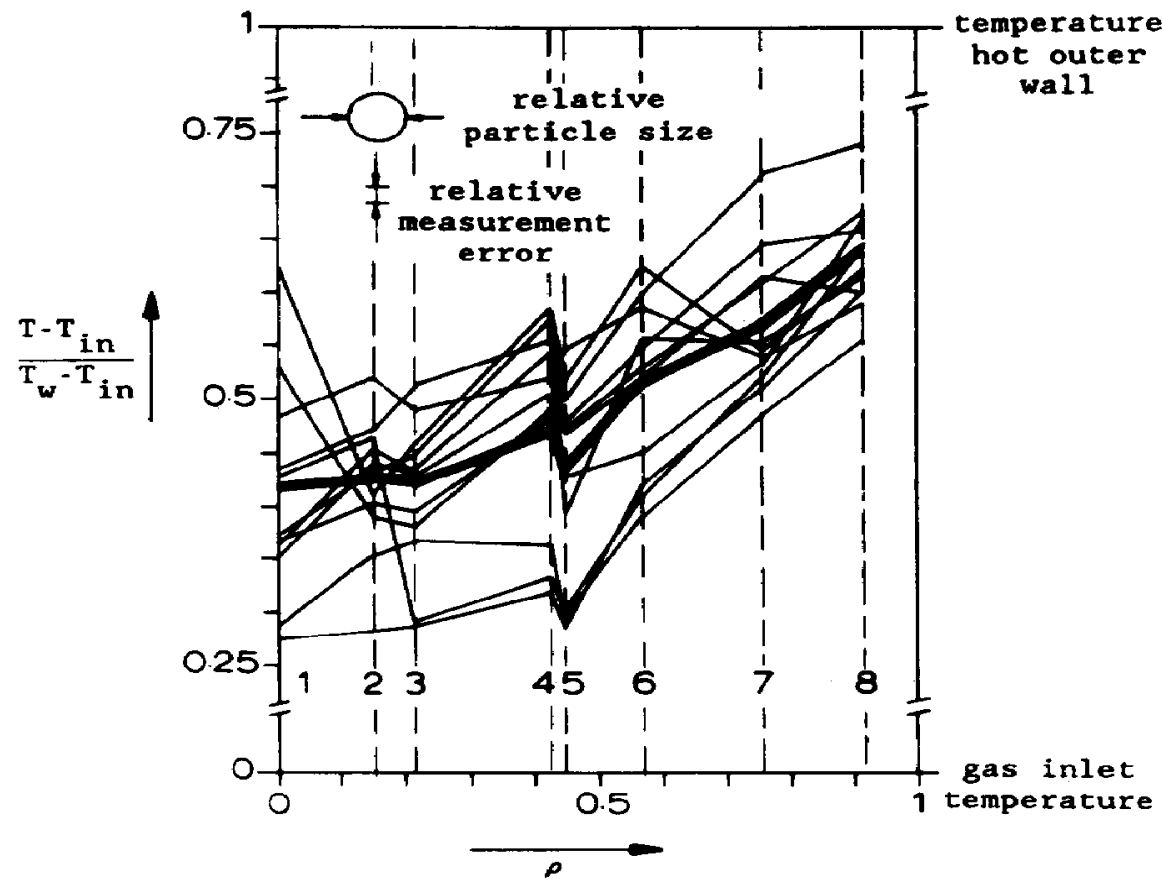

Fig. 3. Measured radial temperature profiles. In between the measurements the bed was fluidized and allowed to settle again. The bold line gives the average profile. Pellet size as indicated.

profile. The profiles were measured with thermocouples stuck into the bed, some 20 particle layers above the bed inlet. The packed bed contained 25 particles on a diameter. Further data: the pellets are Raschig rings with height $\times$ outer diameter $\times$ inner diameter $=8 \times 8 \times 4 \mathrm{~mm}$; cylindrical bed with height $\times$ diameter $=30 \times 20 \mathrm{~cm}$; the gas is air; the superficial velocity is $21 \mathrm{~cm} / \mathrm{s}$.

Notice-that the amount of scattering is considerable. Bed properties, e.g- the effective heat conductivity of the packed bed, $\lambda_{\text {eff }}$, and the heat transfer coefficient at the wall, $\alpha_{w}$, are determined by fitting a model to the measured temperature profiles. The scatter in temperature can induce a scatter in the bed properties like $\lambda_{\text {eff }}$ and $\alpha_{w}$ or a comparable statistical character of the bed. This will be discussed in the next section.

\section{PACKED-BED HEAT TRANSPORT PROPERTIES}

For a cold gas flowing through a packed bed heated at the wall, the temperature as a function of the dimensionless radius, $\rho=r / R_{t}$, and the dimensionless height, $\omega=z / L_{x}$, is given by

$$
\begin{aligned}
\frac{T_{w}-T(\rho, \omega)}{T_{w}-T_{i n}}= & 2 \sum_{i=1}^{\infty}\left\{\frac{J_{1}\left(\lambda_{i}\right) J_{0}\left(\lambda_{i} \rho\right)}{\lambda_{i}\left\langle\left[J_{0}\left(\lambda_{i}\right)\right]^{2}+\left[J_{1}\left(\lambda_{i}\right)\right]^{2}\right\rangle}\right. \\
& \left.\times \exp \left(-\frac{\lambda_{i}^{2} \omega}{P e^{\ominus}}\right)\right\}
\end{aligned}
$$

with $\lambda_{i}$ being the $i$ th root of the equation

$$
B i^{\ominus} J_{0}(\lambda)-\lambda J_{1}(\lambda)=0
$$

and

$$
\begin{gathered}
P e^{\ominus}=\frac{\rho_{e} C_{p, e} v_{0} R_{t}^{2}}{\lambda_{\text {efr }} L_{t}} \\
B i^{\ominus}=\frac{\alpha_{w} R_{t}}{\lambda_{\text {eff }}}
\end{gathered}
$$

[e.g. Lerou and Froment (1978)]. It is assumed that the temperature of the wall of the packed bed is constant over the entire bed length.

By fitting the temperature profiles to the above equation, we determined the effective radial heat conductivity, $\lambda_{\text {eff }}$, and the heat transfer coefficient at the wall, $\alpha_{w}$, as a function of the gas velocity for the packed bed with the temperature profiles as given in Fig. 3. Values of $\lambda_{\text {eff }}$ and $\alpha_{w}$ are obtained by fitting average radial temperature profiles.

In Fig. 4 the results of the fitting procedure are presented for $\lambda_{\text {eff }}$ in the form of $\Gamma=\lambda_{\text {eff }} / \lambda_{\theta}$ with $\lambda_{\theta}$ $=0.037 \mathrm{~W} / \mathrm{m} \mathrm{K}$ vs $R e=v_{0} d_{p} / v_{g}$, with $d_{p}=6 V_{p} / A_{p}$ $=6.1 \mathrm{~mm}$ and $v_{g}=3.3 \times 10^{-5} \mathrm{~m}^{2} / \mathrm{s}$. For $\alpha_{w}$, the results are presented in Fig. 5 in the form of $B i=$ $\alpha_{w} d_{p} / \lambda_{\text {eff }}$ vs $\operatorname{Re}$.

Two sets of data have been obtained. The packed bed was fluidized and allowed to settle again and the radial temperature profile was measured again. Usually, this yielded temperature profiles full of scatter as illustrated in Fig. 3. However, we repeated fluidizing and settling the packed bed over again, until by chance a smooth temperature profile was obtained. For this specific packing we determined $\Gamma$ and $B i$ for several values of $R e$, yielding the full dots. For $\Gamma$ it can be seen from Fig. 4 that the straight-line relation 


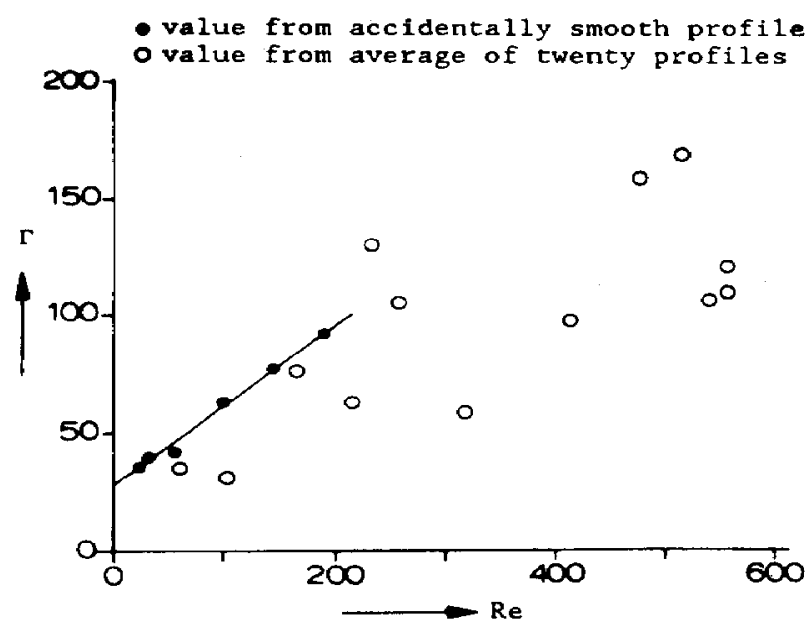

Fig. 4. Dimensionless effective heat conductivity, $\Gamma=$ $\lambda_{\text {eff }} / \lambda_{s}$, vs the particle Reynolds number.

commonly found in the literature is obtained: the intersection with the $\Gamma$-axis gives us the static contribution to $\lambda_{\text {eff }}$ and the slope, the dynamic contribution. Notice that in Fig. 5, for this selected case, the amount of scatter in $B i$ is small. The deviation between the measured and fitted temperature profiles never exceeded $1^{\circ} \mathrm{C}$.

The second set of data are represented by the open dots. For the open dots we fitted the average temperature profile obtained from 20 individual temperature profiles full of scatter. All profiles were measured for one specific gas velocity. For each determination of an individual temperature profile, we fluidized the packed bed and let it resettle. Then we adjusted the gas flow so that the same specific velocity was obtained, and measured the radial temperature profile. Regardless of the amount of scatter, this temperature profile was added to the 19 obtained in a similar way: this gave us one average temperature profile for this specific velocity. This average profile was then fitted to the model. The deviation between the average measured and fitted profiles never exceeded $1^{\circ} \mathrm{C}$. Notice that, if an average profile is fitted, a tremendous amount of scatter is found in the values of $\Gamma$ and $B i$. This scatter is induced by the scatter in the temperature profiles.

\section{DISCUSSION}

The experiments mentioned above lead to the following observations: if we measure $\lambda_{\text {eff }}$ for one single random packed bed, we obtain a very consistent correlation and the results will be highly reproducible for this specific packing. If we alter the packed bed, we will find a new correlation which is just as consistent and reproducible as the first one; nevertheless, it will usually differ from the first one in the parameter values of its correlation equation. This is also consistent with the results reported in the literature. Many authors have reported correlations for $\lambda_{\text {eff }}$ and

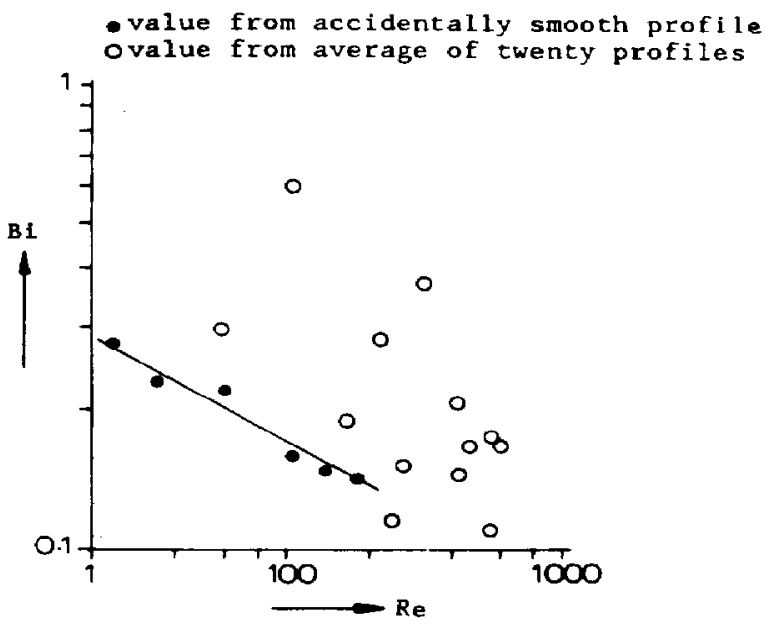

Fig. 5. Biot number, $B i=\alpha_{w} d_{p} / \lambda_{\text {eff }}$, particle Reynolds num-

$\alpha_{w}$ [e.g. Hennecke and Schlünder (1973), Zehner (1973), Zehner and Schlünder (1973), Lerou and Froment (1977), Bauer (1977), Schlünder (1966, 1978) and Dixon and Cresswell (1979)]. The prediction of the properties $\lambda_{\text {eff }}$ and $\alpha_{w}$ by all these correlations differ by a factor of two to four, despite the fact that the authors are known to be accurate, serious and experienced experimentalists. These large discrepancies could be caused in part by the statistical character demonstrated here. Because of the bed-scale statistical behaviour-which, with the same pellets, tubes and gases will only be observed when the experiments are repeated after the bed has been replaced, e.g. by fluidizing and resettling-the probability that different authors find the same correlation is negligible, because the probability to obtain exactly the same random packed bed is negligible. This phenomenon was observed by Gunn and Pryce (1969) for dispersion of mass.

This bed-scale statistical behaviour becomes unimportant if very large packed beds are used with a sufficient number of pellets on a diameter and on a bed height. Regretfully, almost all correlations reported in the literature were obtained from measurements in laboratory set-ups with roughly $10-40$ particles on a diameter: the statistical spread must be considerable, and consequently the literature correlations become less universal.

Even if there would exist a reliable correlation obtained by averaging the results of many randomly poured packed beds, for industrial reactors, the statistical spread could still remain important, e.g. for a cooled tubular reactor with a few particles on a diameter, the value of $\lambda_{\text {eff }}$ would exhibit quite some variation along the tube length. Also, a statistical spread must be expected in the behaviour of different tubes. This would demand correlations not only for $\lambda_{\text {eff }}$ and $\alpha_{w}$, but also for the distributions of both properties. 


\section{CONCLUSIONS}

We observed much scatter in the measured temperature profiles. If $\lambda_{\text {eff }}$ and $\alpha_{w}$ are determined by fitting experimental temperature profiles, the scatter in the profiles will induce a statistical spread in $\lambda_{\text {eff }}$ and $\alpha_{w}$. This spread is considerable and provides an explanation for the large discrepancies between the correlations for $\lambda_{\text {eff }}$ and $\alpha_{w}$ in the literature. Up till now no correlations have been suggested to describe the distributions.

More experimental data, taking into account the statistical character of lumped-bed scale parameters, such as $\lambda_{\text {eff }}$ and $\alpha_{w}$ and pellet-scale parameters, such as $\alpha_{p}$ and $k_{g}$, should be obtained in order to determine general correlations for the distribution of these parameters. In this way the influence of the statistical character on packed-bed operation in industry can be evaluated.

Acknowledgements - The investigations have been and are supported, in part, by the Netherlands Foundation for Chemical Research SON with financial aid from the Netherlands Technology Foundation STW.

\section{NOTATION}

$A_{\mathrm{p}} \quad$ pellet external surface area, $\mathrm{m}^{2}$

$B i$ Biot number based on particle diameter $\left(=\alpha_{w} d_{p} / \lambda_{\text {eff }}\right)$, dimensionless

$C_{p, \theta} \quad$ specific heat of the gas, $\mathrm{J} / \mathrm{kg} \mathrm{K}$

$d_{p}$ particle diameter equivalent to a sphere $\left(=6 V_{p} / A_{p}\right), \mathrm{m}$

$f$ pulsation and rotation frequency of the vortices near the particle contact points, $1 / \mathrm{s}$

$J_{0}, J_{1}$ Bessel functions of the zeroth and first order

$k_{\theta} \quad$ mass transfer coefficient between the particle and the gas, $\mathrm{m} / \mathrm{s}$

$L_{t} \quad$ tube length, length of the packed bed, $m$

$\mathrm{Pe}^{\ominus}$ modified Péclet number based on tube $\left[=\rho_{g} C_{p, g} v_{0} R_{t}^{2} /\left(\lambda_{\text {eff }} L_{t}\right)\right]$, dimensionless

$r \quad$ radial coordinate, $m$

$\operatorname{Re} \quad$ particle Reynolds number $\left(=v_{0} d_{p} / v_{g}\right)$, dimensionless

$R_{t} \quad$ tube radius, $m$

Sr particle Strouhal number $\left(=\varepsilon f d_{p} / v_{0}\right)$, dimensionless

$T \quad$ temperature, $\mathrm{K}$

$T_{\text {in }} \quad$ gas inlet temperature, $\mathrm{K}$

$T_{w} \quad$ temperature of the wall of a packed bed, $K$

$v_{0}$
$V_{p} \quad$ particle volume, $\mathrm{m}^{3}$

$z \quad$ axial coordinate, $m$

\section{Greek letters}

$\alpha_{p} \quad$ heat transfer coefficient between the particle and the gas, $\mathrm{W} / \mathrm{m}^{2} \mathrm{~K}$

$\alpha_{w} \quad$ heat transfer coefficient between the packed bed and the wall, $W / m^{2} \mathrm{~K}$

$\Gamma \quad$ dimensionless effective radial heat conductivity $\left(=\lambda_{\text {eff }} / \lambda_{g}\right)$

$\varepsilon \quad$ porosity at a certain point, being either zero or one, also average bed porosity, dimensionless

$\lambda_{\text {eff }}$ effective radial heat conductivity of the packed bed, W/m K

$\lambda_{g} \quad$ heat conductivity of the gas, $\mathrm{W} / \mathrm{m} \mathrm{K}$

$v_{g} \quad$ kinematic viscosity of the gas, $\mathrm{m}^{2} / \mathrm{s}$

$\rho$ dimensionless radial coordinate $\left(=r / R_{t}\right)$

$\rho_{g}$ density of the gas, $\mathrm{kg} / \mathrm{m}^{3}$

$\omega$ dimensionless axial coordinate $\left(=z / L_{t}\right)$

\section{REFERENCES}

Bauer, R., 1977, Effective radial thermal conductivity of gaspermeated packed beds containing particles of different shape and size distribution. VDI Forschungsh. 582, 39.

Cresswell, D. L., 1986, Heat transfer in packed beds. NATO ASI Ser. Ser. E, 110 (Chem. React. Des. Technol.), 687-728.

Dixon, A. G. and Cresswell, D. L., 1979, Theoretical prediction of effective heat transport parameters in packed beds. A.I.Ch.E. J. 25(4), 663-676.

Hennecke, F. W. and Schlünder, E. U., 1973, Heat transfer in heated or cooled tubes with packings of spheres, cylinders and Raschig rings, Chemie-Ingr-Tech. 45(5), 277-284.

Gunn, D. J. and Pryce, C., 1969, Dispersion in packed beds. Trans. Instn chem. Engrs 47(10), 341-350.

Kirillov, V. A., Matros, Y. S. and Sorokin, V. N., 1972 , Hydrodynamic conditions in the free space of a catalyst bed. Dokl. Akad. Nauk. SSSR 206(6), 1409-1414.

Lerou, J. J. and Froment, G. F., 1977, Velocity, temperature, and conversion profiles in fixed bed catalytic reactors. Chem. Engng Sci. 32(8), 853-861.

Lerou, J. J. and Froment, G. F., 1978, Estimation of heat transfer parameters in packed beds from radial temperature profiles. Chem. Engng $J$. (Lausanne) 15(3), 233-237.

Schlünder, E. U., 1966, Heat and mass transfer in tubes filled with loose material. Chemie-Ingr-Tech. 38(11), 1161-1168.

Schlünder, E. U., 1978, Transport phenomena in packed bed reactors. ACS Symp. Ser. 72 (Chem. React. Engng Rev.Houston), 110-161.

Zehner, P., 1973, Experimental and theoretical determination of the effective heat conductivity of solid beds consisting of spheres with throughflow at moderate and high temperatures. VDI-Forschungsh. 558, 35.

Zehner, P. and Schlünder, E. U., 1973, Effective thermal conductivity of spherical packings perfused at moderate and high temperatures. Chemie-Ingr-Tech. 45(5), 272-276. superficial gas velocity based on empty tube cross-section, $\mathrm{m} / \mathrm{s}$ 